\title{
Unilever food safety assurance system for refined vegetable oils and fats
}

\author{
Gerrit VAN DUIJN \\ Gerrit DEN DEKKER \\ Unilever Supply Chain Technology Unit, \\ P.O. Box 114, \\ 3130 AC Vlaardingen, \\ The Netherlands \\ <Gerrit-van.Duijn@unilever.com>
}

\begin{abstract}
The Unilever Food Safety Assurance system for refined oils and fats is based on risk assessments for the presence of contaminants or pesticide residues in crude oils, and refining process studies to validate the removal of these components. Crude oil risk assessments were carried out by combining supply chain visits, and analyses of the contaminant and pesticide residue levels in a large number of crude oil samples. Contaminants like poly-aromatic hydrocarbons and hydrocarbons of mineral origin, and pesticide residues can largely be removed by refining. For many years, this Food Safety Assurance System has proven to be effective in controlling contaminant levels in refined vegetable oils and fats.
\end{abstract}

Key words: analysis, contaminants, vegetable oils, refining, Unilever
The oil refining process was introduced in 1900 , to improve the quality of oils and fats for application in margarine production, and as cooking oil. The process was optimized to reduce the natural taste and colour, and to remove most of the free fatty acids present in the crude oil. It was discovered years later that under these optimized conditions, the process also reduces the levels of many of the contaminants like metals, poly-aromatic hydrocarbons, and minor components like pesticide residues.

Toward the end of the twentieth century, most of the food companies started a Food Safety Assurance System intended for the ingredients used in their products. Unilever developed a specific system for the supply chains of oils and fats. This system included an assessment for the presence of contaminants and pesticide residues in crude oils and the validation of the refining process for the removal (reduction to below safe limit) of these components:

- the crude oil risk assessment combined visits of all steps of the crude oil supply chains with analyses of contaminants and pesticide residues in crude oils. These analyses were done on all crude oils bought by Unilever for own or toll refining in North West Europe for a period of four years (01/2001-12/2004). The crude oil risk assessment resulted in a crude oil risk matrix;

- oil containing a high level of a specific contaminant or pesticide residue was refined under standard process conditions, and the removal was checked by analyzing the level of this contaminant or pesticide residue in the refined oil.

\section{Crude oil risk assessment}

\section{Supply chain visits}

In the period from January 2003 until July 2007, the supply chains of five major oils used by Unilever were inspected for chemicals use, drying procedures, and housekeeping practices. The following supply chains were assessed: - soybean oil from central Brazil and mid-west USA;

- sunflower oil from South Africa and France;

- rapeseed oil from Germany;

- palm oil from Malaysia (Sabah and peninsular Malaysia) and Indonesia (Sumatra);

- coconut oil from the Philippines.

Farmers, plantation companies, seed storage companies, oil mills, oil transportation, and oil storage companies were visited, and their procedures were examined using questionnaires. Special attention was paid to the following contaminants and chemicals:

- pesticides used during growth and crop protection after harvesting;

- poly-Aromatic Hydrocarbon adsorption from exhaust gases during drying;

- mineral oil from leaking equipments or transport vehicles;

- residues from previous cargoes during transport and storage of crop or crude oil.

The following qualitative observations were done:

Pesticides in oil seeds (for more details on pesticides see van Duijn (2008)

Pesticides are not applied in the last two weeks before harvesting to limit the residue levels in the seeds. Insecticides are sometimes used as post harvest treatment to protect seeds during transport and storage. Carry over of insecticides from previously treated grains may occur in storage silos.

\section{Pesticides in palm oil}

Herbicides are used to control weeds in the circle under the oil palm tree. Contamination of loose fruits by herbicides is minimized by direct application after harvesting. Insects are increasingly controlled by biological pest control; in this case, chemicals are only used to control pest outbreaks. Palm fruits are not stored for long, and therefore postharvest treatment is not applied.

\section{Pesticides in palm kernel and coconut}

Application of pesticides for these crops has not been observed.

\section{Drying practices}

When moisture is too high, oil seeds are dried to a specified water level. This drying can be in direct contact with exhaust gases. This has been observed for soybeans in wood-fired packed bed dryers and sunflower seeds in diesel-fired counter current dryers. Direct dryers are not allowed to be used in Europe and the United States of America.

Drying is an essential operation in the coconut oil supply chain, it avoids Aflatoxin formation and it releases the copra (coconut meat) from the shell. In the method, which is predominant, halved coconuts are dried upside down on a grid of a bamboo over an open fire, burning the coconut shells.

Palm kernels are washed and dried after cracking in the palm oil mill. Most, but not all mills, dry in indirect dryers. 


\section{Mineral oil leakages}

Small, but visible mineral oil leakages have been observed in palm oil mills and at oil seed reception areas. More and more oil mills use food-grade lubricants and hydraulic oils when there is an unavoidable contamination risk.

\section{Residues from previous cargoes:}

Measures were in place in all the visited locations to avoid contamination with previous cargoes. Crop transport from farm or plantation to oil mill was carried out in clean and inspected conveyances. Crude oils were transported from the mill to the port or customer in dedicated and sealed trucks (sealing of empty return trips would further improve security), tank parks were all foodstuff dedicated and overseas transport was according to the previous cargo regulations of the European Union (EU) or Federation of Oils, Seeds and Fats Associations (FOSFA).

\section{Crude oil analyses}

The crude oils bought by Unilever for own or toll refining in North West Europe in the period from January 2001 until December 2004 were analyzed for pesticides, poly-aromatic hydrocarbons (Benz(a)Pyrene) and hydrocarbons (C10-C24). In total, 1595 samples were analyzed, which included crude rapeseed oil (566 samples), crude sunflower oil (154 samples), water-degummed soybean oil (157 samples), crude palm oil (318 samples) crude palm kernel oil (236 samples), and crude coconut oil (164 samples). The samples were taken by an independent superintendent from different levels in the ship tanks and were analyzed in an independent laboratory (Laboratory Dr. A Verweij, Coolhaven 32, 3024 AC Rotterdam, The Netherlands). The results were as follows:

\section{Pesticides}

All samples were analyzed for 24 organochlorine pesticides, 28 organophosphorus pesticides, 9 nitrogen-based pesticides and 4 pyrethroids. The palm oil samples were also analyzed for 14 pesticides frequently used by palm plantations as reported during the supply chain visits. Only a limited selection of mainly organophosphorus insecticides was detectable in crude seed oils (soybean, sunflower and rapeseed oil). Figure 1 gives an overview of the maximum pesticide levels found in rapeseed, sunflower and soybean oil.

No pesticide residues were detected in crude palm oil, palm kernel oil, and coconut oil.

\section{Poly-Aromatic Hydrocarbons}

All crude oil samples were analyzed for Benz(a) Pyrene (BaP) levels. The BaP is generally used as a marker for the presence of Poly-Aromatic Hydrocarbons in crude and refined oils. The EC regulation 1881/2006 (EC, 2006) limits the BaP level in oils and fats intended for direct human consumption or as ingredient in foods at max. 2 ppb.

For the analyses, a sample with a BaP level above $1 \mathrm{ppb}$ was considered to be contaminated. The fraction of contaminated samples was very high for crude coconut oil (79\%), high for crude sunflower oil (12\%) and below $10 \%$ for crude rapeseed oil (9\%), water degummed soybean oil (7\%), and crude palm kernel oil (6\%). None of the palm oil samples had a BaP level above $1 \mathrm{ppb}$. Figure 2 gives an

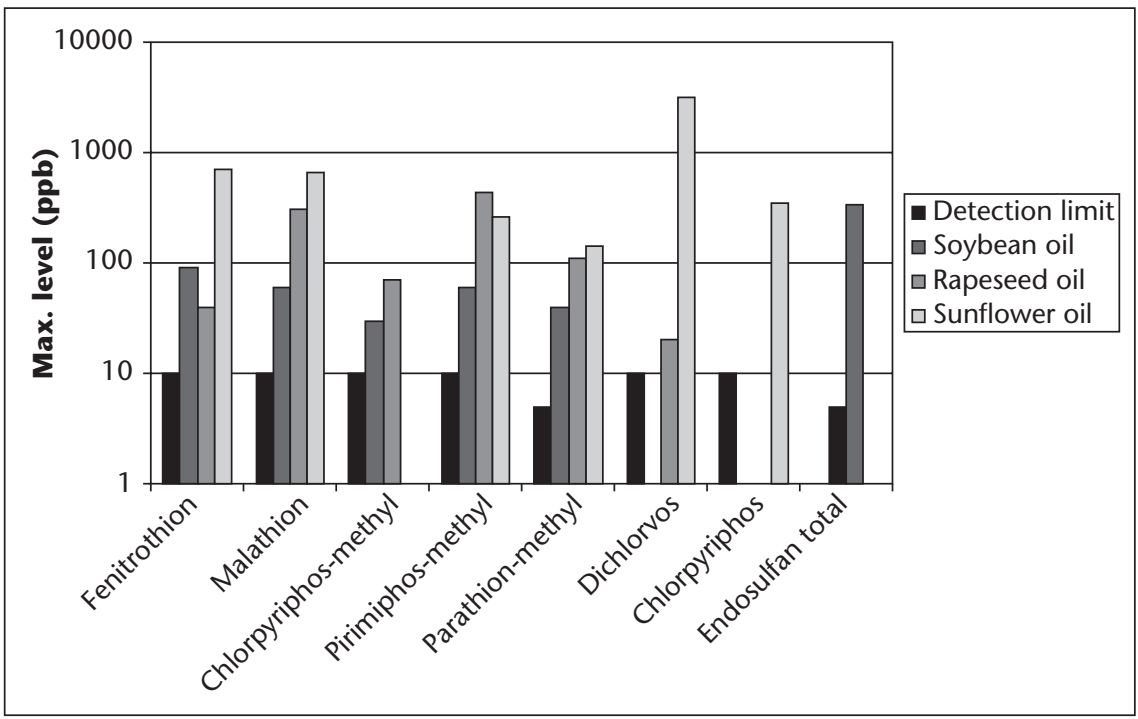

Figure 1. Results of pesticide residue analyses in crude seed oils. This graph shows the detection limit and the maximum observed levels for water-degummed soybean oil, crude rapeseed oil and crude sunflower oil.

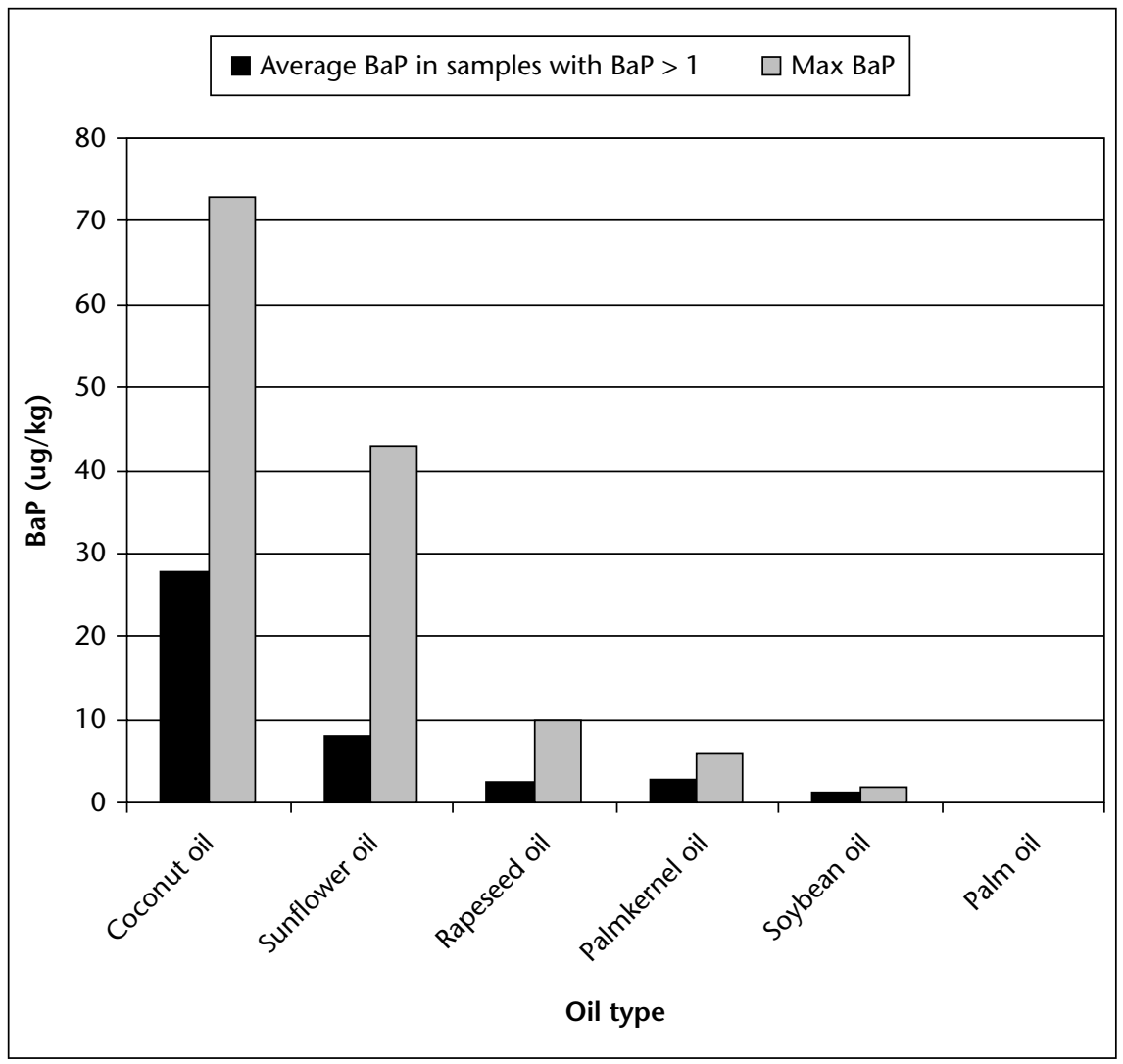

Figure 2. Results of $\mathrm{BaP}$ (Benz(a)Pyrene) analyses in crude oils. This graph shows the average of the samples containing more than 1 ppb BaP and the maximum observed levels. 
overview of the average and maximum BaP levels found in the crude oil samples with a $\mathrm{BaP}$ level higher than $1 \mathrm{ppb}$. This shows that all oils except palm oil may occasionally have a BaP level before refining, which is higher than the EU limit of 2 ppb.

\section{Hydrocarbons}

Hydrocarbons in Mineral oil can be roughly divided in four fractions, depending on the carbon numbers:

- gasoline (C5-C10), purified hexane (C6) is used as a solvent in oil extraction and the residue level in crude oils is restricted in the contracts by the flashpoint $\left(<121^{\circ} \mathrm{C}\right)$;

- kerosene and diesel (C10-C24);

- fuel and lubricant oil (> C16);

- asphalt (> C35).

All tested crude oils were analyzed for kerosene and diesel contamination (C10-C24), since these components were observed to be at a high level in crude palm oil in 1999. Detectable levels of these hydrocarbons were only found in crude palm oil. Figure 3 shows the average and maximum observed levels of hydrocarbons in the range $\mathrm{C} 10-\mathrm{C} 24$ for the years 2001 until 2004. Palm oil also contains natural hydrocarbons in the range of C10-C25; it is not clear so far as to the level of natural hydrocarbons. In 2007, the tri-partnership project of the Malaysian, Indonesian, and Dutch government agreed a maximum level of 25 ppm for C10 C24 hydrocarbons in crude palm oil.

\section{Other contaminants}

The levels of other contaminants like dioxins, PCB's and heavy metals (lead) were monitored in some crude oil and refined oil samples. The levels in none of these samples were above the limits set in EC regulation 1881/2006.

\section{Crude oil risk matrix}

The risk assessment for the presence of a specific contaminant or pesticide residue in crude oil is a combination of the observations made during the supply chain visits and the results of the crude oil analyses. The identified risks are used to establish a crude oil risk matrix. This matrix shows the risk classification (high, medium, low) for presence of a contaminant or pesticide residue in a crude oil, in case the origin of the crude oil is unknown (table 1). The justification of this classification is:

\section{Pesticides}

In total, eight different organophosphorus insecticides were found at detectable levels in seed oils. Crude sunflower oil had the highest maximum observed levels (6 insecticides above $100 \mathrm{ppb}$ of which 1 was higher than $1000 \mathrm{ppb}$ ), crude rapeseed followed (3 insecticides above $100 \mathrm{ppb}$ ), while for soybean oil,

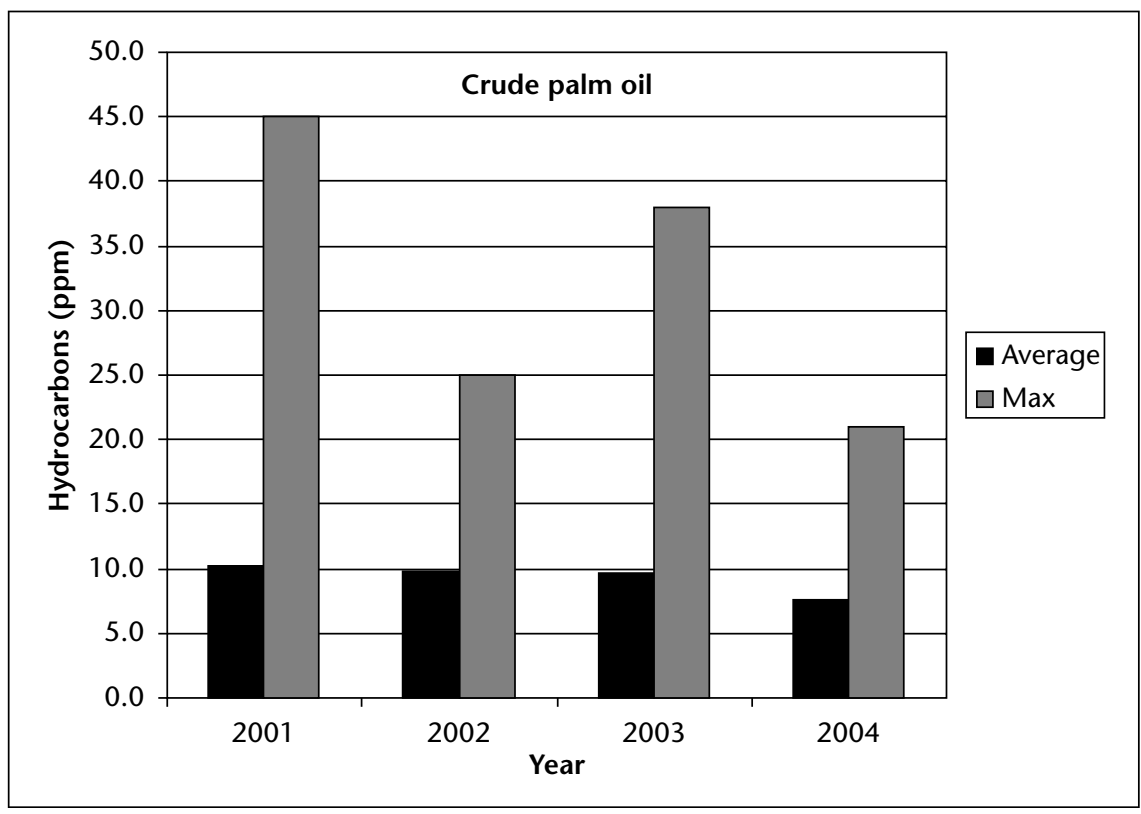

Figure 3. Results of hydrocarbon analyses in crude palm oil. Analyzed were hydrocarbons in the carbon range C10-C24. The graph shows per year the average for all samples and the maximum observed level.

Table 1. The crude oil risk matrix. This matrix shows the risk that a specific contaminant or pesticide residue is present in a crude oil of unknown origin.

\begin{tabular}{|llllll|}
\hline & Pesticides & PAH & $\begin{array}{l}\text { Mineral oil in edible } \\
\text { oil imported in EU }\end{array}$ & $\begin{array}{l}\text { Dioxins and } \\
\text { PCB's }\end{array}$ & $\begin{array}{l}\text { Heavy metals } \\
\text { (lead) }\end{array}$ \\
\hline LIMIT & MRL & BaP $<2 \mathrm{ppb}$ & Fediol CoP & EC/1881/2006 & EC/1881/2006 \\
\hline Soybean oil & Medium & Medium & Low & Low & Low \\
\hline Sunflower oil & High & High & High & Low & Low \\
\hline Rapeseed oil & Medium & Medium & Low & Low & Low \\
\hline Palm oil & Low & Low & High & Low & Low \\
\hline Palm kernel oil & Low & Medium & Medium & Low & Low \\
\hline Coconut oil & Low & Very high & Medium & Low & Low \\
\hline
\end{tabular}

only the maximum observed level for Endosulfan was above $100 \mathrm{ppb}$. Pesticides were not found in the tropical oils (palm, palm kernel, and coconut oil).

\section{Benz(a)Pyrene}

The occurrence and maximum level of $\mathrm{BaP}$ in coconut oil were very high: occurrence $79 \%$, maximum level $73 \mathrm{ppb}$. Sunflower oil had a lower maximum level (43 ppb) and occurrence (12\%). The maximum levels for rapeseed, palm kernel, and soybean oil were between 2 (EC-regulated level) and $10 \mathrm{ppb}$ while the occurrence was below 10\%. No detectable level of $\mathrm{BaP}$ in palm oil has been observed.

\section{Hydrocarbons of mineral oil origin}

Incidents with high levels of hydrocarbons in edible oils have occurred for palm oil (diesel contamination) and sunflower oil (grease oil contamination in 2008). In both the cases, the origins of contamination were never published. The customer cannot be sure if the causes for contamination have been eliminated, therefore the risk of classification remains high. Palm kernel and coconut oil may originate from the same regions as the contaminated palm oil, and are therefore considered as medium risk products.

\section{Other contaminants}

Risks here are classified as low, since; so far, all detected levels are below the EC-regulated limits.

\section{Risk matrix based analyses}

The risk matrix can be used to determine the frequency of the contaminant or pesticide resi- 
due analyses in crude oils from an unknown origin. The proposed frequency is:

- very high and high risk $\rightarrow$ check every delivery;

- medium risk $\rightarrow$ quarterly monitoring;

- low risk $\rightarrow$ annual monitoring

\section{Process validation contaminant or pesticide residue removal}

The following procedure was applied to all crude oils delivered to Unilever, for own or toll refining, with a contaminant or pesticide residue level above detection limit (for hydrocarbons and pesticides) or EC-regulated limit (for BaP):

- the refinery was informed of the contamination of the oil, and the contaminated lot was blocked:

- the refining process was validated for removal of the contaminant or residue level to below detection limit (for hydrocarbons or pesticides) or EC-regulated limit (for $\mathrm{BaP})$. This process validation was done by processing a minimum batch with the standard Unilever recipe for neutralization, bleaching (with active coal for PolyAromatic Hydrocarbon removal), and deodorization and analyzing the contaminant or pesticide level in the processed oil;

- the crude oil was de-blocked and the whole lot was processed when the level of tested deodorized oil was below the limit;

- this process was repeated for every delivery of crude oil with a contaminant or pesticide level higher than the levels of the oils used in previous process validations.

\section{Pesticides}

All pesticides found in the analytical survey were extensively removed to below detection limit under the standard Unilever process conditions (neutralization, bleaching and deodorization at $\mathrm{T}>230^{\circ} \mathrm{C}$ ). Some of the pesticides were already removed during neutralization (e.g., Dichlorvos), others during bleaching (e.g., Pirimiphos-methyl), but all were removed during deodorization because of their volatility. The process conditions of the European refining industry are not so different from the conditions applied by Unilever. Therefore, most of the industrial refineries will reduce organophosphorus pesticides to a level well below the level in the crude oil. However, for each refinery, a process validation is required for pesticide removal.

\section{Poly-Aromatic Hydrocarbons}

Heavy Poly-Aromatic Hydrocarbons (5 rings or more) are removed by active carbon adsorption, while light Poly-Aromatic Hydrocarbons are also removed by stripping at high temperature deodorization $\left(200-240^{\circ} \mathrm{C}\right)$. The $\mathrm{BaP}$ is a heavy Poly-Aromatic Hydrocarbon and needs active carbon treatment for removal.

The Unilever process validation experience shows the following active carbon levels needed to reduce BaP to below $1 \mathrm{ppb}$ :

Coconut oil: $0.19 \%$ carbon for every 10 ppb $\mathrm{BaP}$ in crude oil.

Sunflower and rapeseed oil: $0.17 \%$ carbon for every 10 ppb BaP in crude oil.

A higher active carbon dosage is needed at lower deodorization temperatures. These levels are relatively conservative and result in a reduction to a level well-below EC-regulated limit.

\section{Hydrocarbons of mineral origin}

Process validations were carried out with oils containing less than $25 \mathrm{ppm}$ hydrocarbons in the range $\mathrm{C} 10-\mathrm{C} 24$. High temperature deodorization $\left(\mathrm{T}>230^{\circ} \mathrm{C}\right)$ reduced these hydrocarbons to below detection limit. The reduction of hydrocarbons in the range $\mathrm{C2O}$ C35 was less successful; the volatility of this range is apparently insufficient.

\section{Conclusions}

- The findings of the risk assessment visits correlated very well with the results of the crude oil analyses.
- The pesticides found in crude seed oils are mainly organophosphorus insecticides used to protect oil seeds during storage and transport after harvesting. No pesticides were found in crude palm oil, crude palm kernel oil, and crude coconut oil. Organophosphorus insecticides were extensively removed during refining.

- Poly-Aromatic Hydrocarbons in crude oils originate by absorption from exhaust gases when these are used for direct drying of the oil crop. High levels were found in crude coconut oil since direct drying is a common practice in the coconut oil supply chain. High levels may also occur in crude sunflower oil, while in most other crude oils occasional contaminations may occur. Palm fruits are never dried and Poly-Aromatic Hydrocarbon contamination has not been observed in crude palm oil. Heavy Poly-Aromatic Hydrocarbons are removed by active carbon treatment, while light components will also be reduced by high temperature deodorization.

- Hydrocarbons in the diesel oil range (C10C24) in crude palm oil reduced over the testing period from max. $45 \mathrm{ppm}$ and average 10 ppm to max. 21 ppm and average 8 ppm. Parts of these hydrocarbons are of natural origin. High temperature deodorization $\left(\mathrm{T}>230^{\circ} \mathrm{C}\right.$ ) reduced these hydrocarbons to below detection limit.

- The effectiveness of the Food Safety Assurance System for vegetable oils and fats was confirmed by monitoring of deliveries of industrially refined oils to the Unilever product manufacturing sites.

\section{REFERENCES}

Van Duijn G. Industrial experiences with pesticide removal during edible oil refining. Eur / Lipid Sci Technol 2008; 110: 982-9.

EC commission regulation $N^{0} 1881$. Setting Maximum levels for certain contaminants in foodstuffs. Official Journal of the European Union: L364/5-24, 2006. 